\title{
Research on the Training and Cultivation of Adolescent Football Players' Strength Quality
}

\author{
Zequn Wang ${ }^{1, *}$ Lihong Hou ${ }^{1}$
}

${ }^{1}$ School of Physical Education, Beihua University, Jilin, China

*Corresponding author.

\begin{abstract}
This paper expounds the characteristics, training methods and cultivation ways of adolescent football players, and describes the scientific training of adolescent football players in football training combining with the characteristics of football sports. On the basis of the theory of strength quality, this paper makes a systematic analysis of the main strength quality of adolescent football players by means of experiments and documents, and further explains the importance of strength training for adolescent football players. At the same time, it also lays a deep theoretical foundation for other coaches in the training and cultivation of youth strength.
\end{abstract}

\section{Keywords: football players, strength training, teenagers, training and cultivation}

\section{INTRODUCTION}

Football originated from Chinese Cuju since ancient times, and has been spread to now, and evolved into the current football. With the popularity of football, Federation Internationale de Football Association (FIFA) has held a lot of events, and the participation is also very hot, which causes the active participation of Chinese football players. Since 2002, the Chinese people have always had a dream that they can enter the world cup again, but the result is not satisfactory. The defeat of Chinese football has aroused the deep thought of Chinese people, so there are many kinds of comments on Chinese team from the outside world. Generally speaking, Chinese football should start from the children. First of all, it is necessary to select a child, who is suitable for the development of sports, and then carry out strict training, or even waste the whole study, as well as children's childhood youth. Therefore, it cannot be ignored to lay a good foundation for teenagers' training, especially for the training of strength quality. For football, strength is not only a requirement for one person, but also has great influences on the whole game's tactical formulation, the consumption of physical strength and even the outcome of the game. All these have higher requirements on the physical quality of football players. To play in the game, players must improve their own physical quality, and the strength quality is just the important foundation of physical quality.

\section{A. Experimental subjects}

16 members of football team from Qinhuangdao No.5 Middle School in Hebei Province were selected as the experimental subjects. The 16 students were divided into two groups, with 8 students each group. One group had the training, and was called the experimental group. The other group had no training, and was called the control group. The observation lasted for two months. In this experiment, jumping training, 1-minute sit-ups and solid sphere throwing were used as test data. Sixteen students were tested before the experiment, and then had the retest 2 months later. Finally, comparative data analysis was conducted to obtain the experimental results. The basic information of selected experimental subjects is shown in "Table I".

TABLE I. BASIC INFORMATION LIST OF EXPERIMENTAL SUBJECTS $(\mathrm{N}=16)$

\begin{tabular}{|l|l|l|l|l|}
\hline & \multicolumn{1}{|c|}{ Experimental group } & \multicolumn{1}{|c|}{ Control group } & T & \multicolumn{1}{c|}{ P } \\
\hline Age (years old) & $15.78 \pm 0.45$ & $15.80 \pm 0.35$ & -0.173 & 0.868 \\
\hline Height $(\mathrm{cm})$ & $174.25 \pm 1.98$ & $174.37 \pm 1.77$ & -0.015 & 0.885 \\
\hline Weight $(\mathrm{kg})$ & $63.29 \pm 1.80$ & $63.36 \pm 1.81$ & -2.240 & 0.090 \\
\hline
\end{tabular}

Before the experiment, the indexes of age, height and weight of football players in the experimental group and the control group were tested statistically. The results showed that there was no significant difference $(\mathrm{P}>0.05)$, ensuring the group balance of the experimental objects. 


\section{B. Experimental process}

- Asking the subjects to fill in the informed consent form

- Having the tests before and after the experiment

For standing long jump test, each person had three chances to jump and got the best result.

For solid sphere test, each person had three chances to throw and got the best result.
For 1-minute sit-up test, each person had one chance.

- Training content: 60 minutes per class, training in weeks

- Training time: having training on Monday, Wednesday, Friday and Sunday (lasting for 2 months)

TABLE II. TEST INDEX SCORES BEFORE TRAINING

\begin{tabular}{|l|l|l|l|l|}
\hline \multicolumn{1}{|c|}{ Experimental indicators } & \multicolumn{1}{c|}{ Experimental group } & \multicolumn{1}{c|}{ Control group } & \multicolumn{1}{c|}{ T } & \multicolumn{1}{c|}{ P } \\
\hline Standing long jump (cm) & $252.13 \pm 3.23$ & $256.63 \pm 3.20$ & -2.953 & 0.021 \\
\hline Solid sphere (cm) & $803.88 \pm 24.15$ & $795.13 \pm 21.48$ & 2.546 & 0.038 \\
\hline 1-minute sit-ups (times) & $36.13 \pm 1.73$ & $36.25 \pm 1.68$ & -0.243 & 0.815 \\
\hline
\end{tabular}

It can be concluded from "Table II" that the results of the two groups of athletes before training are relatively similar and belong to normal distribution, which is of experimental significance.

TABLE III. COMPARISON OF TEST INDEXES BEFORE AND AFTER TRAINING

\begin{tabular}{|l|l|l|l|l|l|l|}
\hline \multicolumn{1}{|c|}{$\begin{array}{c}\text { Experimental } \\
\text { indicators }\end{array}$} & \multicolumn{1}{|c|}{$\begin{array}{c}\text { Before the } \\
\text { experiment }\end{array}$} & $\begin{array}{c}\text { After the } \\
\text { experiment } \\
\text { experimental } \\
\text { group) }\end{array}$ & \multicolumn{1}{|c|}{ Variation } & $\begin{array}{c}\text { Before the } \\
\text { experiment }\end{array}$ & $\begin{array}{c}\text { After the } \\
\text { experiment } \\
\text { control group) }\end{array}$ \\
\hline standing long jump (cm) & $252.13 \pm 3.23$ & $267.00 \pm 4.04$ & $14.84 \pm 2.53$ & $256.63 \pm 3.20$ & $256.00 \pm 2.78$ & $0.63 \pm 0.58$ \\
\hline solid sphere (cm) & $803.88 \pm 24.15$ & $1095.50 \pm 86.13$ & $291.62 \pm 66.03$ & $795.13 \pm 21.48$ & $810.63 \pm 33.32$ & $15.50 \pm 24.76$ \\
\hline 1-minute sit-ups (times) & $36.33 \pm 1.73$ & $43.00 \pm 1.85$ & $6.84 \pm 1.45$ & $36.25 \pm 1.68$ & $36.13 \pm 1.25$ \\
\hline
\end{tabular}

The data in "Table III" all belong to normal distribution $(\mathrm{P}<0.05)$. Through the comparison within the group and two months of experimental intervention, the performance of the experimental group has been improved significantly, while the experimental performance of the control group has not been greatly improved, which shows that the training is effective for improving the strength quality of athletes.

\section{STRENGTH CHARACTERISTICS OF FOOTBALL PLAYERS}

\section{A. Strength characteristics of adolescent football players}

Adolescents' ages are a peak period of rapid growth of muscle strength, which is the best opportunity for adolescent football players to develop muscle strength. At this time, adolescents' body will give priority to the development of maximum muscle strength, while the relative strength is not very strong. When the physiological age gradually increases to 17 years old, the relative strength will also increase at this time, so it is necessary to train the strength quality in the critical period of the growth of the strength quality of adolescents.

\section{B. Strength training characteristics of adolescent football players}

According to the development of youth's physical quality and the unique characteristics of football, the special training program is made. In order to meet the training requirements, it is required to carry out comprehensive development training for the strength quality of adolescents, which requires that the training intensity should be close to the intensity of special competitions, and meet the requirements of strength training during competitions, so that the cooperation between muscles can be more coordinated, and their psychological ability can be more full.

In order to achieve the scientific training intensity, it is necessary to make a scientific training plan. Football sports require targeted training, as a skill dominated sports. During this period, the body will produce confrontation. In addition, there is also a great demand for waist, abdomen and lower limb strength. In order to win the advantage in the game, football players should have certain strength and strengthen physical confrontation. At the same time, it lays a deep foundation for the quality of other forces.

\section{The strength of adolescent football players}

1) Upper limb strength: The upper limb strength is often used in football. In the game, the upper limb 
strength will be used to throw long-distance ball out of bounds and conduct physical confrontation, and physical confrontation will also affect the balance of the body, including the flexibility between arms and wrists, which will directly affect the running balance and the ability to reach the ball out of bounds. Therefore, the influence of upper body strength on athletes can't be ignored.

2) Lower limb strength: The strength of the lower limbs is necessary in football. In football, there are often sudden turns and stops. In addition, the sufficient strength of the lower limbs is also an important way to fully maintain physical fitness. Therefore, in the lower limb strength training, it is required to pay more attention to the physical training of the lower limb. Only the combination of the two can lay a good foundation for lower limb strength in adolescence.

3) Waist and abdomen strength: Waist and abdomen strength, also known as core strength, is an important strength quality to maintain human body balance and improve control power. Football players have enough waist and abdomen strength, which can better coordinate the strength of large and small muscle groups and reduce the energy consumption caused by sports during training and competition. And the waist and abdomen strength can be applied to the header attack of football players, and increase the performance of players on the field.

\section{STRENGTH TRAINING METHODS OF ADOLESCENT FOOTBALL PLAYERS}

Strength quality refers to the ability of body muscles to fight against other forces at work. In football, the explosive force, the ability to fight on the field and the core ability of waist and abdomen dominate the training and competition of football players. From the perspective of professional sports training, the strength quality is divided into three types, i.e. maximum strength, fast strength and strength endurance. Each kind of strength has an impact on football players. Therefore, it is necessary to divide the strength quality of adolescent football players into these kinds, and then carry out the strength training methods of step-by-step and individualized teaching.

\section{A. Training methods of fast strength for adolescent football players}

Adolescent football players should cultivate good explosive power. In the game, a football player needs to make a sharp turn, stop, start, and also needs an explosive burst of time to start the ball and change direction suddenly. The player usually needs to complete the above actions in a very short time, which puts forward higher requirements for the fast strength of the football player. In order to complete these quick actions and body changes as soon as possible, it is required to carry out corresponding trainings. In the training of athletes' strength, it is required to carry out different forms of training according to their personal characteristics, gender and age. It is better to pay attention to the development of the strength of lower limb muscles, and the training of active muscles, cooperative muscles, small muscles, large muscles and weak muscles. The explosive force is mainly to make the muscles contract quickly and overcome the resistance, usually using fast and high-frequency practice and super equal length practice. In order to develop the explosive force of adolescent football players, it usually adopts the training mode of high intensity, more groups and less repetitions, and tries to make the way of muscle work consistent with the action of the game. Fast and high-frequency practice can improve the reaction ability and speed awareness of adolescent athletes. Super isometric exercise refers that the muscles first do the concessional work, then stretch, and quickly make the restraint work. Such exercise can turn the strength possessed by individuals into explosive force. Such training usually includes box jumping, step jumping, leapfrog jumping and half squatting with heavy barbell. Such training requirements are usually formulated according to individuals.

\section{B. Training of strength and endurance of adolescent football players}

Strength endurance is also indispensable in football. In modern sports competition, strength endurance has gradually become an important factor in leading the game. Therefore, in football training, teenagers should take strength endurance training as an important training content. The competition of modern football is fierce, the match time is long, and the strength endurance becomes the essential strength quality in the match. In the training of strength and endurance, the training time can be slightly longer than the competition time, and the training intensity can be slightly higher than the competition intensity. In order to combine the explosive force produced in an instant with the endurance with a long duration, the dynamic training method should be selected more for teenagers. However, for the strength endurance training, the static training method should also be selected appropriately, and for the strength endurance training, the barbell squat can be selected. It can also use light weight barbell for multiple strength exercises, and can also carry out long-distance weight-bearing run, so as to develop strength endurance. 


\section{Training of adolescent football players' maximum strength quality}

According to sports physiology, there are mainly two ways to develop the maximum strength of athletes. One is to strengthen the muscle cross section of athletes, and the other is to improve the coordination between muscles. When carrying out the maximum strength training, it is better to improve it from $90 \%$ to $95 \%$ of the maximum bearing capacity of teenagers. After doing a small number of groups, it will recover to the upper and middle strength. This method can ensure the excitability of the neuromuscular system and make the relative strength develop to a certain extent.

\section{PRINCIPLES TO BE NOTICED IN STRENGTH TRAINING OF ADOLESCENT FOOTBALL PLAYERS}

\section{A. Principle of appropriate load}

According to the theory of sports training, it is required to pay attention to the principle of appropriate load in sports training. Coaches should pay attention to the adaptation of human body function according to the actual needs of the players when training the youth football players. In daily training, coaches need to understand all the different changes of athletes at different times and establish the proportion relationship between light and heavy loads. It is better to carry on the strength quality training according to the special project of football sports, so as to enhance the movement speed during the match time. Therefore, it can quickly improve the football sports technology. When training the strength quality of adolescent football players, the load should not be too large, otherwise it will cause fission reaction. It is necessary to take the load intensity corresponding to the body to cause the stress reaction needed by the body, and carry out sports training in a planned and scientific way. ${ }^{1}$

\section{B. Principle of differential treatment}

According to the theory of sports training and the different characteristics of athletes' strength quality, different strength quality training should be carried out for different strength athletes, different training tasks should be formulated, and the corresponding strength load should be arranged. Combined with the special requirements of football, it is an important principle to measure the strength and quality load of football players for the height and weight of each person in each age group. In addition, it is necessary to pay attention to the individual physical quality and technical style of the players, and take different strength training measures according to the individual technical style of the adolescent football players.

\section{Strength training principle of comprehensive development}

Adolescent football players need to adapt to the changing situation of the game, so they need a comprehensive development of strength. When training, they need to train all types of players systematically. In football, it should not only train the strength of lower limbs, but also the strength of upper limbs, so as to strengthen the competition. In strength training, it should not neglect the training of core strength of athletes or the training of large muscle group strength. At the same time, it is better to pay attention to the training of small muscle group, so as to avoid the sports injury in the future sports, and the development of muscle group can fully improve the coordination ability, the enthusiasm and response ability for the competition.

\section{Recovery principle of excessive strength}

When training adolescent strength, it is necessary to pay attention to the recovery of strength. The overrecovery does not refer to the ordinary recovery, but refers to the next training when the athlete's body is not fully recovered. Such training helps adolescent better lay a good foundation for their physical quality, which is conducive to the continuous increase of adolescents strength.

\section{E. Principle of combination of strength training and football specific technology}

In order to ensure the continuity and accuracy of football, it is better to pay attention to the combination of football application skills with the training of adolescents' strength quality. When athletes develop muscle strength quality, football technology should also reach the corresponding level. With special systematic training, the characteristics of muscle strength should be consistent with the game. Through the accumulation, the quality of football special strength is transferred to the technical structure of football, and the sports technology is strengthened.

\section{WAYS TO CULTIVATE THE STRENGTH QUALITY OF ADOLESCENT FOOTBALL PLAYERS}

\section{A. Making a training plan for the strength quality of adolescent football players}

Adolescent body is in an important stage of rapid growth. If coaches want to make their strength level adapt to the level of competition, it is necessary to make their strength training intensity consistent with the competition requirements, which requires the coaches to develop a set of training plan suitable for different age groups. Also, the coaches should carry on strict and scientific systematic training for the adolescents. It can take targeted training, so as to avoid 
some of the blindness and randomness in training. At the same time, the coaches should increase the athletes' interest in training according to the athletes' hobbies and their own characteristics, and a good training plan can greatly reduce the trouble of sports injury.

\section{B. Strengthening the training of all aspects of quality}

It should carry out all-round training on the strength and physical quality of teenagers, and lay a good foundation for their physical quality from childhood. At ordinary times, it should also strengthen the training of speed, endurance, flexibility, sensitivity and coordination. After having these physical qualities, athletes can have a great improvement on the basis of their original strength, so that adolescent athletes can easily pass the platform period of strength growth and reduce unnecessary sports injuries during training.

\section{Strengthening the training of psychological quality}

Training an excellent football player is not only in physical quality, but also in psychological quality. It is necessary to let the athletes understand that the training of strength quality is to make them better carry out high-level confrontation, so it is required to guide the athletes to have correct motivation. According to the theory of sports training, it is necessary to stimulate the individual motivation of athletes to participate in training. It is required to make athletes overcome difficulties and guide them to engage in strength quality training. It should also make athletes understand the purpose of training, improve their interest in training, and change their training from being passive to being active. In addition to motivation, willpower is also necessary. Long-term sports training cannot be separated from the persistence of athletes. The willpower of excellent athletes should be better than that of ordinary people. For strength training, it is often necessary to adhere to the last to reflect its proper training effect. In addition, it is also required to strengthen the ability to cultivate the self-confidence of adolescent football players. Many adolescents are prone to fatigue in strength training, which leads to sports injury. After recovery, athletes often have fear of difficulties. Therefore, it is necessary to cultivate adolescents' self-confidence in time, help adolescent football players overcome psychological obstacles, so as to improve sports performance.

\section{Importance of venue facilities and coaches}

Football has high requirements for facilities. A good venue can enhance the training interest of players, reduce sports injuries, improve sports performance, and lay a solid foundation for future training. Football training is naturally inseparable from coaches. Excellent coaches will train in a planned and scientific way and update their professional knowledge in time to meet the requirements in the new situation. Their basic theoretical knowledge and professional skills are very solid. Only such coaches can make football players lay a solid foundation in their youth, so as to raise the level of Chinese football to a new level as a whole.

\section{CONCLUSION}

It's a hard, long and special process to train the strength quality of teenagers. Teenagers are in the main stage of physical development, and there should be special training methods for them. Different from person to person, it is necessary to make gradual progress and promote personal all-round development, grasp the sensitive period of strength growth to carry out strength quality training, and reduce the training that affects the physical development and sports injury, such as long-term static training, over weight squatting and drop jump. During the training period, it is required to take the athletes as the main body, fully consider the psychological characteristics and physical development characteristics of the athletes, and make the corresponding training plan, which has an important impact on the psychological and physical health of the athletes.

\section{References}

[1] Wang Pengtao. Research on the cultivation of the reserve force of Japanese football [J]. Liaoning Sport Science and Technology, 2003(02): 17-19. (in Chinese)

[2] Pan Ye. On the Importance of Strength Training for Juvenile Football Players [J]. The Science Education Article Collects (Late Journal), 2012(03): 138-139. (in Chinese)

[3] Yang Chuanxi. On the development strategies of strength training of adolescent football $[\mathrm{J}]$. Contemporary Sports Technology, 2014, 4(10): 40+42. (in Chinese)

[4] Zhang Zhaohui, Wang Qian. On the cultivation of speed and strength quality of adolescent football [J]. Public Literature and Art, 2010(15): 44. (in Chinese)

[5] Dong Qi. Study on the strength training structure of adolescent football players in China [J]. Journal of Hubei University of Economics (Humanities and Social Sciences Edition), 2012, 9(03): 219-220. (in Chinese)

[6] Fan Xiang. Research on the strength training of adolescent basketball players [J]. Sports Teachers, 2019, 42(04): 25-26. (in Chinese)

[7] Zou Julu. Strength training of adolescent football should not be prematurely adultized [J]. Physical Teachers, 2007(01): 42-43. (in Chinese)

[8] Zhu Wenjun. Physical education is an effective way to cultivate students' willpower [C]. Western physical education research, 2014, No. 4 (total, No. 136). Gansu Institute of Physical Education Science, 2014: 83-85. (in Chinese)

[9] Zhu Kongyu. Analysis on the current strength training of high school sports students in China $[\mathrm{J}]$. Contemporary Sports Science and Technology, 2017, 7(01): 36-37. (in Chinese)

[10] Qi Xianshuang, Hou Lihong. On the cultivation and training of adolescent basketball players in China [J]. Modern Communication, 2014(04): 125-126. (in Chinese)

[11] Sports Training [M]. People's Sports Press, Tian Maijiu, 2000. 